\title{
Vom Suizid zu seiner Verhütung
}

Patrick Haemmerle

Dr. med., Vorstandsmitglied der GGSP, Mitglied FMH

Aus gegebenem Anlass - einem bevorstehenden Symposium zum Thema (s.u.), sowie den derzeit laufenden Arbeiten für einen nationalen Aktionsplan Suizidprävention - lohnt es sich, einen kurzen Blick auf die Geschichte des Suizides und seiner Verhütung zu werfen.

Verschiedene geschichtliche Dokumente, inklusive der Bibel, belegen, dass Suizid eine menschliche Handlung ist, die seit Urzeiten existiert und praktiziert wurde und auch heute weiterhin präsent ist. Deutlich weniger ergiebig verläuft indessen die Suche nach den Ursprüngen und Beweggründen der Suizidverhütung. Im Folgenden sollen einige Eckdaten und einige Gedanken zur Geschichte der Suizidprävention referiert werden. Für weiterführende Informationen werden einschlägige Publikationen aufgeführt und wird vor allem auf mehrere aktuelle Veranstaltungen hingewiesen.

\section{David und Saul}

Auf der Suche nach frühen Zeugnissen von Bemühungen zur Verhütung einer Selbsttötung stossen wir auf die biblische Geschichte von David und Saul.

In der Tat können wir Davids Zitherspiel, um Saul von seinem «bösen Geist» zu befreien (wir müssen heute annehmen, dass es sich um eine melancholische, bzw. depressive Krankheit handelte), als frühe musiktherapeutische Intervention begreifen. Diese scheint vorübergehend, aber leider nicht dauerhaft, gewirkt zu

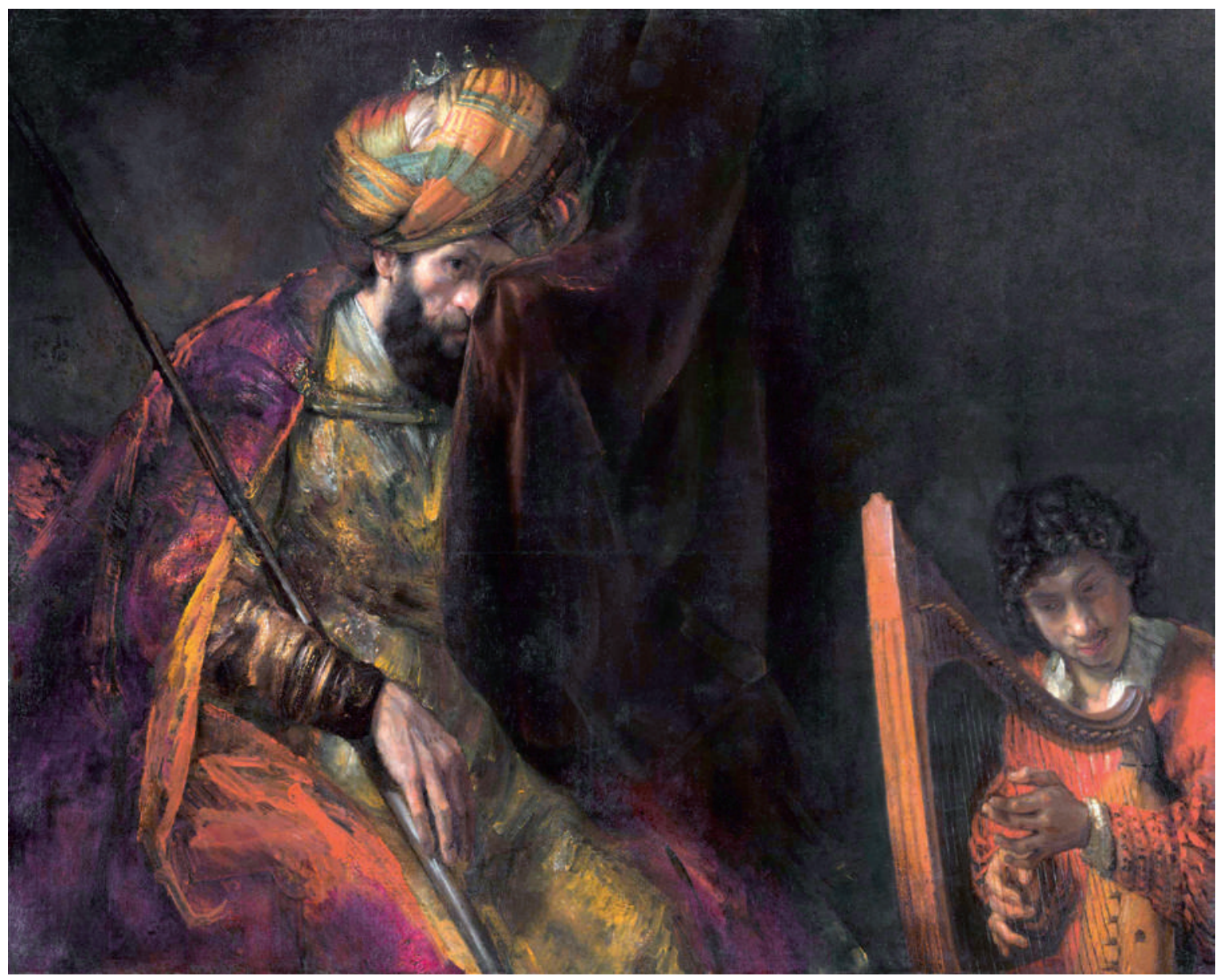

Rembrandt (1606-1669): David spielt die Harfe für Saul (1657); Gemälde im Museum Mauritshuis Den Haag («So oft nun ein Geist Gottes Saul überfiel, nahm David die Zither und spielte darauf. Dann fühlte sich Saul erleichtert, es ging ihm wieder gut, und der böse Geist wich von ihm.» 1 Sam 16, 14-24 und 18, 10-12). 
haben, da wir beim Weiterlesen der Geschichte ja erfahren, dass sich Saul zu einem späteren Zeitpunkt dennoch in sein Schwert stürzte und Suizid beging.

\section{Kirche deklarierte Selbstmord zur schweren Sünde}

Während weder der später sich ereignende Suizid von Saul noch eine der weiteren neun in der Bibel berichteten Selbsttötungen (vgl. Ebo Aebischer, 1999) mit ablehnendem oder gar verurteilendem Kommentar berichtet werden, kam es unter dem Kirchenvater Augustinus dann zu einer massiven Verurteilung dieses Aktes: Die Selbsttötung wurde zur schweren Sünde deklariert, zum «Selbstmord» eben, der Sünde «wider den Heiligen Geist», die in der Folge von der Kirche massiv geächtet und sanktioniert wurde. In der Tat wurden den Leichnamen der von eigener Hand gestorbenen Menschen nicht nur das Begräbnis auf dem geweihten Gottesacker verweigert, sondern diese Leichname wurden erneut gerichtet und zum Tode verurteilt, und, post mortem, gevierteilt, verbrannt oder erhängt. Wenn wir zwar davon ausgehen können, dass der Leichnam diese Torturen nicht mehr wahrnehmen konnte, so müssen die zusätzlichen Strafmassnahmen aus heutiger Sicht doch als äusserst bedenklich gewertet werden: In der Tat wurden in der Folge die Güter des Verstorbenen eingezogen, wodurch ganze Familien in himmeltrauriges Elend gestürzt wurden. Wenngleich mit erheblichem Vorbehalt, müssen wir diese massiven kirchlichen (Verweigerung des Begräbnisses) und später auch weltlichen (eben der Einzug der Güter der betroffenen Familie) Sanktionen einer Selbsttötung als frühe, präventiv intendierte Massnahmen verstehen: Verhinderung durch Vergällung und Abschreckung. Wie wir heute wissen, war diese "präventive Strategie» nicht von Erfolg gekrönt: Suizid ist nicht mit Abschreckung beizukommen!

Die weltliche Verurteilung des Suizides fand, zumindest im nach-revolutionären Frankreich, 1791, per Dekret, ein Ende. Dem Individuum wurde vermehrt Selbstbestimmung zugestanden, wodurch Selbsttötung nicht mehr geächtet werden konnte. In der katholischen Kirche indessen wurden erst im Jahre 1983 die Regeln des kirchlichen Rechtes geändert, so dass seither auch Menschen, die sich das Leben genommen hatten, in "gesegneter Erde» begraben werden durften.

\section{Aderlass und Ehe als «Therapie»}

Es wirkt aus heutiger Sicht eher skurril, wenn wir einige suizidpräventive Interventionen im 19. Jahrhundert zur Kenntnis nehmen: So waren die «médecins aliénistes», die sog. "Irrenärzte», also eigentlich die Vorgänger der späteren Psychiater, überzeugt, dass bei Männern ein schlichter Aderlass manchmal genügen konnte, um die suizidalen Ideen des Individuums zu zerstreuen. Für junge Frauen wiederum wurde die Ehe als Therapeutikum empfohlen (Monestier, 1995). Die Ärzte dieser Periode fanden weitere, vor allem auf den Körper orientierte Methoden, um die Betroffenen von ihren suizidalen Gedanken und Akten abzuhalten.

Eine eigentliche organisierte Suizidprävention entstand erst im frühen 20. Jahrhundert. Diese frühen Ansätze setzten v.a. auf das «Zuhören». Die allererste Initiative dieser Art machte Pfarrer Warren, der 1885 für eine Pfarrei in New York zuständig war. Sein Neffe, ebenfalls Pastor, schuf 1906 in Los Angeles ein eigentliches "Zuhör-Zentrum», das auch heute noch funktionieren soll.

Die erste europäische Initiative fand in England statt, wo 1935 das erste Zentrum eröffnet wurde, unter der Leitung von Chad Varah, dem Rektor der anglikanischen Kirche in einem Stadtbezirk von London. Der Erfolg war überwältigend und diese Initiative wurde bald zur «Gesellschaft der Samariter" (The Samaritans), die weiterhin existiert und heute mehrere Tausende freiwillig Mitarbeitende zählen soll. Gemäss dem englischen Modell wurden bald darauf in Belgien (Télé-Accueil), in Bali (Avec Vous), in Berlin (Hilfe für Lebensmüde), in New York (Dargebotene Hand) weitere entsprechende Zentren eingerichtet. Später folgten die Schweiz, Italien, Finnland, Österreich usw. (Monestier, 1995).

Ein weltweit erstes Zentrum mit dem deklarierten Ziel der Suizidprävention wurde 1948 von Erwin Ringel, einem Pionier der suizidpräventiven Intervention, geschaffen. 1960 veranlasste diese wichtige Persönlichkeit die Gründung der Internationalen Gesellschaft für Suizidprävention, International Association for Suicide Prevention (IASP), deren erster Vorsitzender er wurde (Monestier, 1995).

\section{Suizidprävention in der Schweiz}

In der Schweiz wurden suizidpräventive Bemühungen vor allem im Rahmen der sogenannten PsychohygieneBewegung propagiert. Entsprechende Vereine entstanden in der Mitte des letzten Jahrhunderts in der Westschweiz (Sociétés d'hygiène mentale) und in der Deutschschweiz.

Ausgehend von der sog. «Gøtland-Studie» - gleichsam der «Mutter der Suizidpräventionsprogramme in Europa» (Wolfgang Rutz et al., 1992) - führte die FMH ab 1992 eine landesweit konzipierte Weiter- und Fortbildungsveranstaltung für Allgemein- und Hausärzte durch, die diesen erlauben sollte, depressive und suizi- 
dale Patientinnen und Patienten frühzeitig zu erkennen und zu behandeln (FMH, 1992).

Während der ebenfalls in den 90er Jahren gegründeten Schweizerischen Gesellschaft für Krisenintervention und Suizidprophylaxe, SGKS, kein dauerhafter Erfolg beschieden war - übrigens ebenso wenig wie der CAPS (Coordination et action pour la prévention du suicide) in der Westschweiz -, wurde 2003 schliesslich IPSILON gegründet, die Initiative für die Prävention von Suizid in der Schweiz, als Dachorganisation aller in der Schweiz tätigen Vereine, Vereinigungen und Instanzen zur Verhütung von Suiziden.

Ab dem gleichen Jahr entstanden in mehreren Kantonen (u.a. in ZG, BS, GE, SG) die sog. «Bündnisse gegen Depression" - Vereine, die sich an einem in Deutschland entwickelten, multi-sektoriellen und Mehr-Ebenen-Modell orientierten, d.h. mit dem Einbezug möglichst vieler Berufsgruppen und Lebensbereiche, wie Gesundheit, Soziales, Erziehung, Polizei, Rettungswesen, Kirchen usw., das letztlich doch auch auf Suizidprävention ausgerichtet ist, auch wenn der weniger explizite, mehr auf einen der wichtigsten Risikofaktoren für suizidale Handlungen - die Depression - fokussierende Titel gewählt wurde.

\section{Assistierter Suizid}

Seit einiger Zeit wird die Diskussion um die Suizidprävention stark vom Thema des assistierten Suizids beeinflusst. Dies drängt sich auf, wenn entsprechend tätige Organisationen, wie Exit und Dignitas, für sich in Anspruch nehmen, ebenfalls Suizidprävention zu betreiben - eine Behauptung, die explizit suizidpräventiven Vereinigungen leicht «in den falschen Hals» gerät. Nichtsdestoweniger haben die Aktivitäten beider Ansätze - des suizidpräventiven und des sterbebegleitenden - zur Folge, dass die Art und der Zeitpunkt unseres Sterbens zu einem intensiv diskutierten Thema geworden sind.

Überhaupt ist Suizid und Suizidprävention derzeit ein «angesagtes Thema». Dies nicht etwa «nur» wegen einiger Suizide von bekannten Persönlichkeiten in der letzten Zeit, und auch nicht nur wegen der soeben angedeuteten Spannungen in diesem Felde, sondern insbesondere deswegen, weil der Bund derzeit einen Nationalen Aktionsplan Suizidprävention in Ausarbeitung hat. Ausgelöst von einer Motion in der Herbstsession 2013 (Motion Ingold Maja, 11.3973: Suizidprävention - Handlungsspielraum wirkungsvoller nutzen), welche der Bundesrat entgegennehmen musste, wurde - nach einer ersten Fachtagung im letzten Herbst, anlässlich derselben die Verantwortlichen des BAG einen ersten Entwurf präsentierten - in diesem

\section{Veranstaltungshinweise}

Nachdem, wie erwähnt, einiges zur Geschichte des Suizides publiziert worden ist, gilt es die Geschichte der Suizidprävention noch zu schreiben. Aus diesem Grunde hat sich die GGSP (Gesellschaft für die Geschichte der Schweizer Psychiatrie) entschlossen, ihr diesjähriges Jahres-Symposium dieser Thematik zu widmen. Dieses Symposium findet am 25. August 2016 in Zürich statt: «Zur Geschichte des Suizids und seiner Prävention». Das Programm finden Sie unter der Rubrik "Aktuelles» der GGSP: www.ggsp.ch

Bereits am 13. Juni fand in Lausanne, am medizinhistorischen Institut, IUHMSP, ein ganztägiger Anlass statt - «La pathologie du suicide - pour une nouvelle histoire des enjeux médicaux et sociopolitiques aux $19^{\mathrm{e}}$ et $20^{\mathrm{e}}$ siècles" -, dessen Referate zu gegebener Zeit publiziert werden sollen.

Ausserdem finden anlässlich des jährlich stattfindenden Internationalen Tages zur Suizidprävention, dem World Suicide Prevention Day, WSPD (www.iasp.info/wspd), vom 10. September, diverse Veranstaltungen statt, von der Ostschweiz bis Genf: Diese können auf der Homepage von IPSILON (www.ipsilon.ch/de/aktuell/agenda.cfm) gefunden werden. Zudem führt das "Forum Suizidprävention» auch dieses Jahr wieder eineTagung durch, nämlich das 6 . Forum Suizidprävention (im Alter) zum Thema: «Prävention des assistierten Suizids (Sterbehilfe)», am Montag, den 31.10.2016 im Gottlieb Duttweiler Institut in Rüschlikon (Programm abrufbar unter: www.ipsilon.ch/de/aktuell/agenda.cfm).

Frühjahr eine breite Vernehmlassung des Projektes durchgeführt. Das beauftragte Bundesamt analysiert derzeit die Antworten und sollte gegen den Herbst hin einen umsetzbaren Entwurf zuhanden der Exekutive vorbereiten, der dann vom Parlament verabschiedet und ab 2017 zur Umsetzung gelangen sollte.

\section{Disclosure statement}

Der Autor Patrick Haemmerle, Dr. med., MPH, KJPP FMH, gibt folgende ideelle Interessenverbindungen an: Er ist Vorstandsmitglied von IPSILON und GGSP, Präsident des Vereins Suizidprävention des Kantons FR und lebhaft daran interessiert, dass der geplante Nationale Aktionsplan Suizidprävention erfolgreich umgesetzt werden wird.

\section{Ausgewählte Literatur}

- Aebischer-Crettol Ebo, 1999: Aus zwei Booten wird ein Floss. Suizid und Todessehnsucht: Erklärungsmodelle, Prävention und Begleitung. Haffmanns Sachbuch, Zürich.

- FMH, 1992: Krise und Suizid. Basisdokument der Seminare für Aerzte. Bern.

- Hegerl Ulrich et al., 2003: Optimierte Versorgung depressiver Patienten und Suizidprävention: Ergebnisse des «Nürnberger Bündnisses gegen Depression». Dtsch Arztebl 2003; 100(42).

- Minois Georges, 1996: Geschichte des Selbstmordes. Artemis \& Winkler, Zürich

- Monestier Martin, 1995: Suicides. Histoire, techniques et bizarreries de la mort volontaire. Des Origines à nos jours. Le cherche midi éditeur, Paris.

- Rutz W. et al., 1992: Long-term effects of an educational program for general practitioners given by the Swedish Committee for the Prevention and Treatment of Depression. Acta Psychiatr Scand 1992;85:

83-88.

\section{Bildnachweis}

https://de.wikipedia.org/wiki/Saul\#/media/File:Rembrandt_Harmensz._van_Rijn_030.jpg 